\title{
Efficient procedure for the evaluation of multiple scattering and multiphonon corrections in inelastic neutron-scattering experiments
}

\author{
J. Dawidowski \\ Comisión Nacional de Energía Atómica and CONICET, Centro Atómico Bariloche, (8400) San Carlos de Bariloche, \\ Río Negro, Argentina \\ F. J. Bermejo \\ Instituto de Estructura de la Materia, Consejo Superior de Investigaciones Científicas, Serrano 123, E-28006 Madrid, Spain \\ J. R. Granada \\ Comisión Nacional de Energía Atómica and CONICET, Centro Atómico Bariloche, (8400) San Carlos de Bariloche, \\ Río Negro, Argentina \\ (Received 26 January 1998)
}

\begin{abstract}
We present a full set of procedures to evaluate the experimental corrections needed to derive physically meaningful quantities from the measured neutron intensities in inelastic neutron-scattering experiments. Multiple-scattering corrections are evaluated by means of a Monte Carlo code, in which a combination of experimental data and the Synthetic Model is used to account for neutron-molecule interactions. Multiphonon corrections are treated with an iterative scheme. To illustrate the procedure the densities of vibrational states of deuterated water and ice near room temperature are evaluated from data measured in a chopper spectrometer.
\end{abstract} [S0163-1829(98)00326-9]

\section{INTRODUCTION}

Neutron scattering is, presently, a well-established tool to investigate the spatiotemporal correlations in condensed matter. It is superior to other radiation-scattering techniques because of the simplicity of the thermal-neutron nucleus interaction, which enables the measurement of the dynamic correlations over a wide zone of the energy-momentum space. Being an intensity-limited technique, most of the efforts devoted to its optimization during several decades regarded the increase in usable neutron fluxes or in the increase in resolution in energy and/or momentum transfers without a concomitant loss in intensity that is too severe. On the contrary, most of the efforts regarding an improvement of the capabilities of the technique by means of efficient data handling, reduction, and correction procedures have only recently started to gain momentum, mainly as a result of some active international collaborations. ${ }^{1}$

However, the optimal use of the technique requires that the quantities measured as neutron counts are transformed into other physically meaningful magnitudes that are expressed on an absolute scale. Such a step, or rather a series of steps have, over the years, proven to be substantially difficult, even if the probe interaction with the sample is that simple. The referred corrections arise from the unavoidable fact that the samples to be measured are of finite width and that, in very many cases, they have to be contained within some device.

Neutron scattering has a good number of advantages over other techniques in many fields, such as in the investigation of structural or magnetic excitations in disordered solids. Contrary to other techniques presently available, measurements can be performed on dynamic phenomena involving energy transfers between some tens of nanoelectronvolts up to electronvolts. To do this, a wide range of neutronscattering spectrometers are today available, from which one can perform high resolution detailed studies of a particular region, or a wide energy spectrum to cover all the ranges of possible vibrational excitations. This allows an almost direct access to densities of vibrational states without the difficulties presented in the case of optical experiments due to an unknown coupling coefficient. ${ }^{2}$ Nevertheless, the analysis of experimental data to obtain the desired density of states has to pass through several stages that make the process not so straightforward, but which can be successfully completed if previous knowledge of some properties of the material under study is available. These steps can be summarized as: (a) subtraction of the scattering from the empty cell; (b) correction for multiple-scattering effects; (c) correction of singlescattering data by the attenuation factor; (d) subtraction of multiphonon effects.

The container scattering contribution to the observed spectrum can be important not only in the case where the container is necessarily thick (such as in high-pressure measurements), but also where the signal due to the sample in some particular region of the spectrum is weak. Normally empty-cell runs are performed to subtract its contribution from the observed spectrum. Although this subtraction cannot be directly made, a procedure devised by Paalman and Pings ${ }^{3}$ borrowed from $\mathrm{x}$-ray techniques is normally employed. However, the attenuation factors may sometimes strongly depend on the neutron energy via the total cross section, and even if this effect is correctly accounted for, there could be higher-order cell-sample scattering processes that this procedure fails to describe.

The multiple-scattering correction is a long debated subject, and an extensive bibliography can be found, ranging from the pioneering theoretical works of Sears, ${ }^{4}$ to the nu- 
merical simulations made by Copley. ${ }^{5}$ The former, while providing a solid basis for understanding the process, can only be applied to certain simple geometries, ${ }^{6}$ and its main simplifications hinder its application to cases such as tripleaxis spectrometers, where a high degree of detail of the multiple processes is required. That is the reason why the approach through full numerical simulations as proposed by Copley is the best one to treat this problem.

Once the multiple-scattering corrections are performed, the remaining single-scattering component has to be corrected by attenuation of the neutron beam inside the sample, which is dependent on the sample geometry and the neutron energy. Again, in this case, corrections as made by Paalman and Pings are usual, although they fail to account for the full effect of the change in the neutron's mean-free path due to the exchange of energy with the sample. Another important subject not always taken into account is the effect due to the efficiency of the detectors, which is a function of the detected neutron energy. A careful analysis of this fact, combined with the multiple-scattering effect, leads to the conclusion that singly and multiply scattered neutrons are detected with different effective efficiencies because of the different energy distributions (of the neutrons) resulting from such processes. This reasoning is also valid in the analysis of different samples and temperatures, which leads us to the conclusion that corrections by detector efficiency are a sampledependent effect.

Through Monte Carlo simulations all the abovementioned problems can be fully considered, provided that an adequate model to represent the neutron energy and its angular dependence is used. The Synthetic Model was introduced over a decade ago, ${ }^{7}$ aiming to describe the incoherent interaction of neutrons with molecular systems using a minimum set of input data to model the molecular vibrations, which are represented as Einstein oscillators. A scrutiny of its predictive capabilities showed that it provides an adequate description of many integral magnitudes of the doubledifferential cross sections like total cross sections, angular distributions, and inelasticity corrections needed for diffraction experiments, as well as other magnitudes of fundamental interest in nuclear engineering. ${ }^{8}$ Recently, it was applied in a Monte Carlo scheme to calculate inelasticity corrections and multiple-scattering contributions in a two-axis reactor experiment on light and heavy water. ${ }^{9,10}$ The main advantage of this model is that it provides a way to obtain analytical expressions for the required distributions that can be further employed as input for a Monte Carlo evaluation of contributions such as multiple scattering. It is therefore of considerable help in devising scattering kernels for further scattering calculations which, most of the time have been the result of tedious guesswork.

After the above-mentioned effects are properly accounted for and subtracted from the experimental data, they still contain multiphonon effects, which can only be minimized by lowering the temperature, a procedure which is not desirable if temperature effects are investigated. Iterative procedures are normally employed to correct for this effect. In the case of a coherent scatterer, a correction scheme was recently published, ${ }^{11}$ that provides a self-consistent calculation of single and multiple phonon effects.

In this paper we introduce a set of correction procedures to account for the above-mentioned effects in the particular case of an inelastic neutron-scattering experiment, and extend the range of applicability of a procedure already reported on neutron-diffraction experiments. ${ }^{9,10}$ To simultaneously account for multiple scattering, beam attenuation, and efficiency effects, a Monte Carlo simulation procedure is presented that employs as input the uncorrected experimentally observed distributions extended with the synthetic model when data out of the experimental kinematic range are required. This scheme is presented with the addition of an iterative correction procedure, and its relationship with the program MSCAT Ref. 5 is discussed. Finally, an iterative procedure to correct multiphonon effects is described. The analysis is applied to an experiment on deuterated water and ice near room temperature performed at spectrometer MARI (ISIS pulsed neutron source, Rutherford Appleton Laboratory, U.K.), a preliminary report of which was given elsewhere. ${ }^{12}$

\section{MULTIPLE-SCATTERING CALCULATIONS}

\section{A. Definitions}

In order to introduce the basic equations, we will first focus our attention in a case where the sample dimensions are vanishingly small compared to the neutron's mean-free path for any energy in the range of interest, so that only single-scattering processes take place. A monatomic sample will be assumed, but a generalization to multiatom species is straightforward. All further developments aim at the specific analysis of an inelastic-scattering experiment, with a monochromatic incident neutron beam of wave vector $\mathbf{k}_{0}$.

We will suppose a sample without any preferential direction, so we can make an average over all directions. Let $S(Q, \omega)$ be the scattering law of the sample $E_{0}$, and $E$ the incident and final neutron energy, $\mathbf{k}_{0}$ and $\mathbf{k}$ the initial and the final neutron wave vectors, respectively, $d \Omega$ the element of solid angle in the emergent neutron direction, and $\sigma_{b}$ the bound-atom scattering cross section. Then, the double differential cross section defined as the average number of neutrons scattered with final energies between $E$ and $E+d E$, and within a solid angle $d \Omega$, per unit incident flux is ${ }^{13}$

$$
\frac{d^{2} \sigma}{d \Omega d E}=\frac{N \sigma_{b}}{4 \pi} \frac{k}{k_{0}} S(Q, \omega),
$$

where $N$ is the total number of scattering centers. A normalization condition for this expression can readily be found,

$$
\sigma\left(E_{0}\right)=\frac{N \sigma_{b}}{4 \pi} \int \frac{k}{k_{0}} S(Q, \omega) d \Omega d E,
$$

where $\sigma\left(E_{0}\right)$ is the total scattering cross section at the incident energy. As usual, we define $\mathbf{Q}=\mathbf{k}_{0}-\mathbf{k}$ and $\hbar \omega=E_{0}$ $-E$, so we can write

$$
\varepsilon=\hbar \omega=-\frac{\hbar^{2} Q^{2}}{2 m}+\frac{\hbar^{2}}{m} k_{0} Q \mu,
$$

in which $m$ is the neutron mass, $\mu=\cos \theta$, and $\theta$ is the angle between vectors $\mathbf{k}_{0}$ and $\mathbf{Q}$. The double integration in Eq. (2) can be performed with respect to $Q$ according to the relation 


$$
k d \Omega d E=\frac{\hbar^{2}}{m} d^{3} Q,
$$

where

$$
d^{3} Q=2 \pi Q^{2} d Q d \mu,
$$

so taking $Q$ and $\varepsilon$ as variables

$$
k d \Omega d E=\frac{2 \pi}{k_{0}} Q d Q d \varepsilon,
$$

Eq. (2) results in

$$
\sigma\left(E_{0}\right)=\frac{N \sigma_{b}}{2 k_{0}^{2}} \int_{0}^{\infty} Q d Q \int_{\varepsilon_{\min }}^{\varepsilon_{\max }} S(Q, \varepsilon) d \varepsilon,
$$

where $\varepsilon_{\max }$ and $\varepsilon_{\min }$ indicate the allowed kinematic range

$$
\varepsilon_{\text {max }}=\frac{\hbar^{2} k_{0} Q}{m}\left(1-\frac{Q}{2 k_{0}}\right) ; \quad \varepsilon_{\text {min }}=-\frac{\hbar^{2} k_{0} Q}{m}\left(1+\frac{Q}{2 k_{0}}\right) .
$$

Equation (7) establishes the total cross section as the normalization condition of the scattering law, making explicit reference to the kinematic range.

Let us now turn to the case of a sample of finite size, with the addition that it can be enclosed in a container. Let $A$ be the cross-sectional area of the sample, perpendicular to the incident beam. It is convenient to define, following Sears, ${ }^{4}$ a new magnitude, the double differential macroscopic cross section, as the probability that an incident neutron with a wave vector $\mathbf{k}_{0}$ will emerge from the sample-container set with a wave vector $\mathbf{k}$. In this definition we do not take into account neutrons noninteracting with the sample (i.e., transmitted). Its expression thus reads

$$
\frac{d^{2} \Sigma}{d \Omega d E}=\frac{N \sigma_{b}}{4 \pi A} \frac{k}{k_{0}} s(Q, \varepsilon) .
$$

In this expression $s(Q, \varepsilon)$ is the same as $s\left(\mathbf{k}_{0}, \mathbf{k}\right)$ proposed by Sears, an effective scattering function. Here $\hbar Q$ is the total momentum interchange and $\varepsilon$ the total-energy exchange of the neutron with the sample after an unknown number of interactions. The identification of the variables $(Q, \varepsilon)$ with $\left(\mathbf{k}_{0}, \mathbf{k}\right)$ is possible because a monochromatic incident beam is assumed. The effective scattering function admits a decomposition, in part due to neutrons scattered once in the sample $s_{1}(Q, \varepsilon)$, another due to scattering once in the can $s_{C}(Q, \varepsilon)$, and a third due to multiply scattered neutrons (with any combination of sample-can scattering events) $s_{M}(Q, \varepsilon)$ :

$$
s(Q, \varepsilon)=s_{1}(Q, \varepsilon)+s_{M}(Q, \varepsilon)+s_{C}(Q, \varepsilon) .
$$

We now need to define the transmission factor for a neutron of energy $E$ at position $\mathbf{r}$ inside the sample, flying in a direction given by the unit vector $\hat{\mathbf{k}}$, which has to traverse a distance $x$ to emerge from the sample, as the probability that the neutron will not interact in that distance

$$
\mathcal{T}(E, \mathbf{r}, \hat{\mathbf{k}}, x)=\exp \left(-\int_{0}^{x} \Sigma_{t}(E, \mathbf{r}+\xi \hat{\mathbf{k}}) d \xi\right),
$$

where $\Sigma_{t}(E, \mathbf{r})$ is the macroscopic neutron total cross section for an energy $E$, at position $\mathbf{r}$. Here it is understood that different materials can be part of the sample, in order to describe either an inhomogeneous sample or a samplecontainer set. The integral of Eq. (11) over all the sample surface exposed to the incident beam is the sample total transmission $\mathcal{T}\left(E_{0}\right)$.

The value of $s_{1}(Q, \varepsilon)$, defined in Eq. (10), is simply related to $S(Q, \varepsilon)$ through

$$
s_{1}(Q, \varepsilon)=S(Q, \varepsilon) H(Q, \varepsilon),
$$

where $H(Q, \varepsilon)$ is the attenuation factor, i.e., the fraction of lost single scattered neutrons due to multiple scattering and absorption, which can be expressed as the volume integral

$$
\left.H(Q, \varepsilon)=\frac{1}{V} \int d \mathbf{r} \mathcal{T}\left[E_{0}, \mathbf{r}, \hat{\mathbf{k}}_{0}, L\left(\mathbf{r},-\hat{\mathbf{k}}_{0}\right)\right] \mathcal{T} E, \mathbf{r}, \hat{\mathbf{k}}, L(\mathbf{r}, \hat{\mathbf{k}})\right],
$$

where $L(\mathbf{r}, \hat{\mathbf{k}})$ is the distance from $\mathbf{r}$ to the sample surface along $\hat{\mathbf{k}}$.

The normalization condition for Eq. (9) is the fraction of neutrons interacting with the sample

$$
1-\mathcal{T}\left(E_{0}\right)=\frac{N \sigma_{b}}{2 A k_{0}^{2}} \int_{0}^{\infty} Q d Q \int_{\varepsilon_{\min }}^{\varepsilon_{\max }} s(Q, \varepsilon) d \varepsilon,
$$

which corresponds to Eq. (7) for a real experiment.

We will now find a relation of these magnitudes with the typical experimental output dataset, which can be obtained from a time-of-flight inelastic neutron-scattering experiment, in regular intervals of $Q$ and $\varepsilon$, after performing a background subtraction. Let us call it $\mathcal{E}(Q, \varepsilon)$. If we perform the integration of Eq. (7), we define the normalization constant $\alpha$

$$
\alpha=\int_{0}^{\infty} Q d Q \int_{\varepsilon_{\min }}^{\varepsilon_{\max }} \mathcal{E}(Q, \varepsilon) d \varepsilon .
$$

Comparison with Eq. (14), allows us to obtain a simple relationship between the $s(Q, \varepsilon)$ and the experimental data

$$
s(Q, \varepsilon)=\frac{2 A k_{0}^{2}\left[1-\mathcal{T}\left(E_{0}\right)\right]}{\alpha N \sigma_{b}} \mathcal{E}(Q, \varepsilon),
$$

so the experimental components [i.e., single scattered neutrons $\mathcal{E}_{1}(Q, \varepsilon)$, neutrons scattered in the can $\mathcal{E}_{C}(Q, \varepsilon)$, and multiply scattered neutrons $\left.\mathcal{E}_{M}(Q, \varepsilon)\right]$ have the same relationship with the magnitudes defined in Eq. (10). In the following sections we will describe the procedure to subtract $s_{M}(Q, \varepsilon)$ and $s_{C}(Q, \varepsilon)$ from $s(Q, \varepsilon)$, and to correct by the attenuation factor [Eq. (12)], to obtain $S(Q, \omega)$.

\section{B. The Synthetic Model}

The Synthetic Model $^{7}$ allows us to describe the scattering law through a simple expression $T\left(Q, \omega ; E_{0}\right)$ in which $E_{0}$ acts as a parameter. It was devised originally for molecular gases, but it proved to work well for liquids and polymers. It is based on a description whose main features are (a) The translational motion of the molecular unit is that corresponding to an ideal gas of particles of mass equal to the molecular mass, or that of a set of molecules to represent the collective 
behavior. (b) No coupling between internal degrees of freedom is considered. (c) The vibrational spectrum is represented by Einstein oscillators. (d) Effective temperatures and masses are defined, that vary with the incident energy $E_{0}$, according to the internal modes that can be excited by the neutron.

Although this model is intended for incoherent scattering only, the energy distributions that can be calculated from it can represent fairly well the behavior of a coherent scatterer (although not in the low- $Q$ limit), as evidenced by the excellent results obtained for inelasticity corrections in diffraction experiments on heavy water. ${ }^{8}$ We will employ it to calculate multiple-scattering effects, for which structural features are averaged out in their contribution to the angular distributions.

\section{Correction procedure}

The general outline of the Monte Carlo procedure employed in this paper to correct for multiple scattering and attenuation effects, is similar to the method proposed by Copley, ${ }^{5}$ so we will override a detailed description, giving instead a schematic overview. An iterative procedure is proposed in which angle and energy distributions are taken from the experimental data in a first run. Its output is taken as input in the next run until no variations in the multiplescattering components are observed. First we will describe how neutron histories are built, and then how they are scored and recorded.

\section{Neutron histories}

Neutron histories are followed individually, and the flight paths of each step are obtained randomly from the distribution defined by the mean-free paths of the materials that the neutron has to traverse at the current energy $E$, to emerge from the sample. For this purpose, macroscopic cross sections for the sample and can materials are tabulated as input. The probability distributions for the flight paths are altered in order that the neutron never gets out of the sample. Thus, the probability that a neutron of energy $E$ that is in position $\mathbf{r}$, flying in direction $\hat{\mathbf{k}}$, will interact after a distance $x$ is

$$
p(E, \mathbf{r}, \hat{\mathbf{k}}, x)=\frac{\Sigma_{t}(E, \mathbf{r}+x \hat{\mathbf{k}}) \mathcal{T}(E, \mathbf{r}, \hat{\mathbf{k}}, x)}{1-\mathcal{T}[E, \mathbf{r}, \hat{\mathbf{k}}, L(\mathbf{r}, \hat{\mathbf{k}})]},
$$

where the denominator is the mentioned alteration. To compensate for this alteration, a weight is assigned to each neutron that decreases according to the transmitted fractions in the traversed paths, 1 being the initial value. Given the weight at step $i-1$, the weight at step $i$ is calculated as

$$
w_{i}=w_{i-1}\{1-\mathcal{T}[E, \mathbf{r}, \hat{\mathbf{k}}, L(\mathbf{r}, \hat{\mathbf{k}})]\} \frac{\Sigma_{s}(E, \mathbf{r})}{\Sigma_{t}(E, \mathbf{r})},
$$

where $\Sigma_{s}(E, \mathbf{r})$ and $\Sigma_{t}(E, \mathbf{r})$ are the macroscopic scattering and total cross sections, respectively, and its ratio indicates the probability that the neutron will not be absorbed in the considered path. A history is finished when the weight drops under a predetermined cutoff value.
For the assignment of new energies and flight directions, we employ the following criteria, depending on whether the scattering event takes place in the container or in the sample:

(a) Scattering in the container. Elastic scattering is assumed, and the angular distributions are taken from the empty can experimental data.

(b) Scattering in the sample. In this case we treat differently the cases of the first and the subsequent scattering events. If it is the first scattering, the experimental distributions are employed. According to them, the (normalized) probability that a neutron will be scattered with final energy between $E$ and $E+d E$, and in the solid angle $d \Omega$ is [cf. Eqs. (9), (14)-(16)]

$$
\begin{aligned}
P_{\text {exp }}\left(E_{0}, E, \theta\right) & =\frac{1}{1-\mathcal{T}\left(E_{0}\right)} \frac{d^{2} \Sigma}{d \Omega d E} \\
& =\frac{N \sigma_{b}}{4 \pi A\left[1-\mathcal{T}\left(E_{0}\right)\right]} \frac{k}{k_{0}} S_{\text {exp }}(Q, \varepsilon),
\end{aligned}
$$

where $S_{\text {exp }}(Q, \varepsilon)$ is $s(Q, \varepsilon)$ at the starting point of the iterative scheme described below.

For the subsequent scattering events, the distributions are taken from the synthetic model. This allows us a faster computation of the multiple processes, while avoiding the difficulty of the modification of the kinematic range as a function of the varying neutron energy, so the corresponding probability will be

$$
P_{S M}\left(E_{0}, E, \theta\right)=\frac{N \sigma_{b}}{4 \pi \sigma\left(E_{0}\right)} \frac{k}{k_{0}} T\left(Q, \omega ; E_{0}\right) .
$$

\section{Scoring}

At each step, the contribution of the current history to the final spectrum is scored for a set of detectors spanning the desired angular range. In order to calculate the energy bin in which the contribution takes place, we consider the time of flight necessary for a neutron of final energy $E$ to reach the detector in the case of single scattering

$$
T_{s}=\sqrt{\frac{m}{2}} \frac{L_{s}}{\sqrt{E}}=\sqrt{\frac{m}{2}} \frac{L_{s}}{\sqrt{E_{0}-\varepsilon}},
$$

where $L_{s}$ is the flight path from the sample to the detector. If we consider a case of a multiple-scattering process of order $N$, where a path $L_{i}$ is traversed inside the sample with an energy $E_{i}$, then the time of flight will be

$$
T_{s}=\sqrt{\frac{m}{2}}\left(\sum_{i=1}^{N} \frac{L_{i}}{\sqrt{E_{i}}}+\frac{L_{s}}{\sqrt{E_{s}}}\right) .
$$

This process will be regarded in the final spectrum as an equivalent single-scattering event in which the effective energy transfer is $\varepsilon_{e f f}$, so Eq. (21) is verified,

$$
T_{s}^{\prime}=\sqrt{\frac{m}{2}} \frac{L_{s}}{\sqrt{E_{0}-\varepsilon_{e f f}}} .
$$

Therefore the effective energy transfer bin $\varepsilon_{e f f}$, the final energy $E$ that has to be computed in a multiple-scattering 
process in which successive flight paths $L_{i}$ are traversed with corresponding intermediate energies $E_{i}$, is

$$
E=\left(\sum_{i=1}^{N} \frac{L_{i} / L_{s}}{\sqrt{E_{i}}}-\frac{1}{\sqrt{E_{0}-\varepsilon_{e f f}}}\right)^{-2},
$$

and (the cosine of) the angle $\phi$ where the detector is placed, which has to be computed to contribute to the $Q_{\text {eff }}$ bin, will be

$$
\cos \phi=\frac{2 E_{0}-\varepsilon_{e f f}-\frac{\hbar^{2}}{2 m} Q_{e f f}^{2}}{2 \sqrt{E_{0}\left(E_{0}-\varepsilon_{e f f}\right)}} .
$$

We will now focus our attention on the quantity to be scored. For a neutron that at step $i$ is in the sample or in the can with a wave vector $\mathbf{k}_{i}$, for a given effective energy transfer $\varepsilon_{e f f}$ and an effective impulse transfer $\hbar Q_{e f f}$, for which we suppose a detector placed in a direction defined by the angle calculated in Eq. (25), this quantity is

$$
z_{i}\left(\varepsilon_{e f f}, Q_{e f f}\right)=w_{i} P_{e x p}\left(E_{i}, E, \theta\right) \mathcal{T}\left(E, \mathbf{r}_{i}, \hat{\mathbf{k}}\right) C(E) .
$$

The final energy $E$ is computed according to Eq. (24), and the emerging angle $\phi$ only for detectors placed in a symmetry plane with axis in the incident beam. For scoring purposes we use the probability $P_{\text {exp }}\left(E_{i-1}, E, \theta\right)$ taken from Eq. (19) for every order of scattering. Angle $\theta$ is between $\mathbf{k}_{i}$ and the emerging direction, and the detector efficiency $C(E)$ is included to compute the effective number of detected neutrons. Finally, the transmission factor referred to in Eq. (11) is included. It is interesting to consider which is the average after a large number of histories of $z_{1}\left(\varepsilon_{e f f}, Q_{e f f}\right)$, i.e., for single-scattering processes. This is easy to calculate, taking into account that Eq. (26) has to be weighted with the probability of occurrence of an interaction at position $\mathbf{r}$ for an incident neutron of wave vector $\mathbf{k}_{0}$ that is given by Eq. (17) $p\left[E_{0}, \mathbf{r}, \hat{\mathbf{k}}_{0}, L\left(\mathbf{r},-\hat{\mathbf{k}}_{0}\right)\right]$. The result of such an average is

$$
\left\langle z_{1}\right\rangle=\frac{N \sigma_{b}}{4 \pi A\left[1-\mathcal{T}\left(E_{0}\right)\right]} \frac{k}{k_{0}} S_{\text {exp }}(Q, \varepsilon) H^{\prime}(Q, \varepsilon),
$$

where we have introduced the modified attenuation factor

$$
\begin{aligned}
H^{\prime}(Q, \varepsilon)= & \frac{1}{V} \int d \mathbf{r} \mathcal{T}\left[E_{0}, \mathbf{r}, \hat{\mathbf{k}}_{0}, L\left(\mathbf{r},-\hat{\mathbf{k}}_{0}\right)\right] \\
& \times \mathcal{T}[E, \mathbf{r}, \hat{\mathbf{k}}, L(\mathbf{r}, \hat{\mathbf{k}})] C(E) .
\end{aligned}
$$

This attenuation factor, which has to be compared with that given in Eq. (13), includes the detector efficiency, and also takes account of the attenuation in the container.

\section{Iterative procedure}

The proposed iterative procedure starts with the raw experimental data $\mathcal{E}^{(0)}(Q, \varepsilon)$ as input (although it also contains multiple and can scattering components), to provide a first guess of $P_{\text {exp }}\left(E_{0}, E, \theta\right)$, where the superscript indicates that the data is corrected after $n$ iterations. From the first Monte Carlo run we can define the single-scattering ratio as

$$
f_{M S}^{(1)}(Q, \varepsilon)=\frac{s_{1}^{(1)}(Q, \varepsilon)}{s_{1}^{(1)}(Q, \varepsilon)+s_{C}^{(1)}(Q, \varepsilon)+s_{M}^{(1)}(Q, \varepsilon)},
$$

and the attenuation factor $H^{\prime}(Q, \varepsilon)$ is obtained from Eq. (27), where the $S(Q, \varepsilon)$ is obtained from the input data through Eq. (16), thus resulting in

$$
H^{\prime(1)}(Q, \varepsilon)=\frac{\alpha N \sigma_{b}}{2 A k_{0}^{2}\left[1-\mathcal{T}\left(E_{0}\right)\right]} \frac{s_{1}^{(0)}(Q, \varepsilon)}{\mathcal{E}^{(0)}(Q, \varepsilon)} .
$$

The iterative process is devised to correct the experimental data by these two factors, so at the end of iteration $i$ we obtain

$$
\mathcal{E}^{(i+1)}(Q, \varepsilon)=\frac{f_{M S}^{(i)}(Q, \varepsilon) \mathcal{E}^{(0)}(Q, \varepsilon)}{H^{\prime(i)}(Q, \varepsilon)} .
$$

The reason for correcting multiple scattering and can effects through a factor rather than by direct subtraction results from the possibility of obtaining negative values in the first iterations that would invalidate the iterative procedure. The correction factors are recalculated at each step. The expression that results from Eq. (31) has to be renormalized via Eq. (15), and the probability resulting from Eq. (19) is used as input for the next iteration. The iterative process continues until no significant differences in the multiple scattering and can contributions are observed between iterations. It can be easily proved that when this condition is met, the factor correction method converges to the simple subtraction of multiple scattering and can effects. So, finally, the last values of multiple and can scattering are subtracted from the initial experimental data, and the final attenuation factor is applied to obtain the desired $S(Q, \omega)$, as shown in Eqs. (10) and (12).

\section{Multiphonon corrections}

We present an iterative correction method that is based on Ref. 11, where a general outline of it was given. The starting point is the phonon expansion performed on the incoherent approximation for a molecular system of mass $M$,

$$
S(Q, \omega)=e^{-2 W} \sum_{j=1}^{\infty} \frac{1}{j !}\left(\frac{\hbar^{2} Q^{2}}{2 M}\right)^{j} u_{j}(Q, \varepsilon),
$$

where $W$ is the Debye-Waller factor, $u_{j}(Q, \varepsilon)$ are the $j$-phonon functions, with

$$
u_{1}(Q, \varepsilon)=\frac{Z(\varepsilon)}{\varepsilon}(n(\varepsilon)+1),
$$

$Z(\varepsilon)$ is the density of states, and the $j$ th term is obtained through the recursion relation

$$
u_{j}(Q, \varepsilon)=\int_{-\infty}^{\infty} d \varepsilon^{\prime} u_{j-1}\left(Q, \varepsilon-\varepsilon^{\prime}\right) u_{1}\left(Q, \varepsilon^{\prime}\right),
$$

where $n(\varepsilon)$ is the occupation number. It is worth noticing that the $Q$ dependence of $u_{j}$, although not explicitly expressed in Eq. (33), is due to the coherent effects, and it is rigorously valid only for incoherent scattering. The present prescription makes use of the incoherent approximation, 

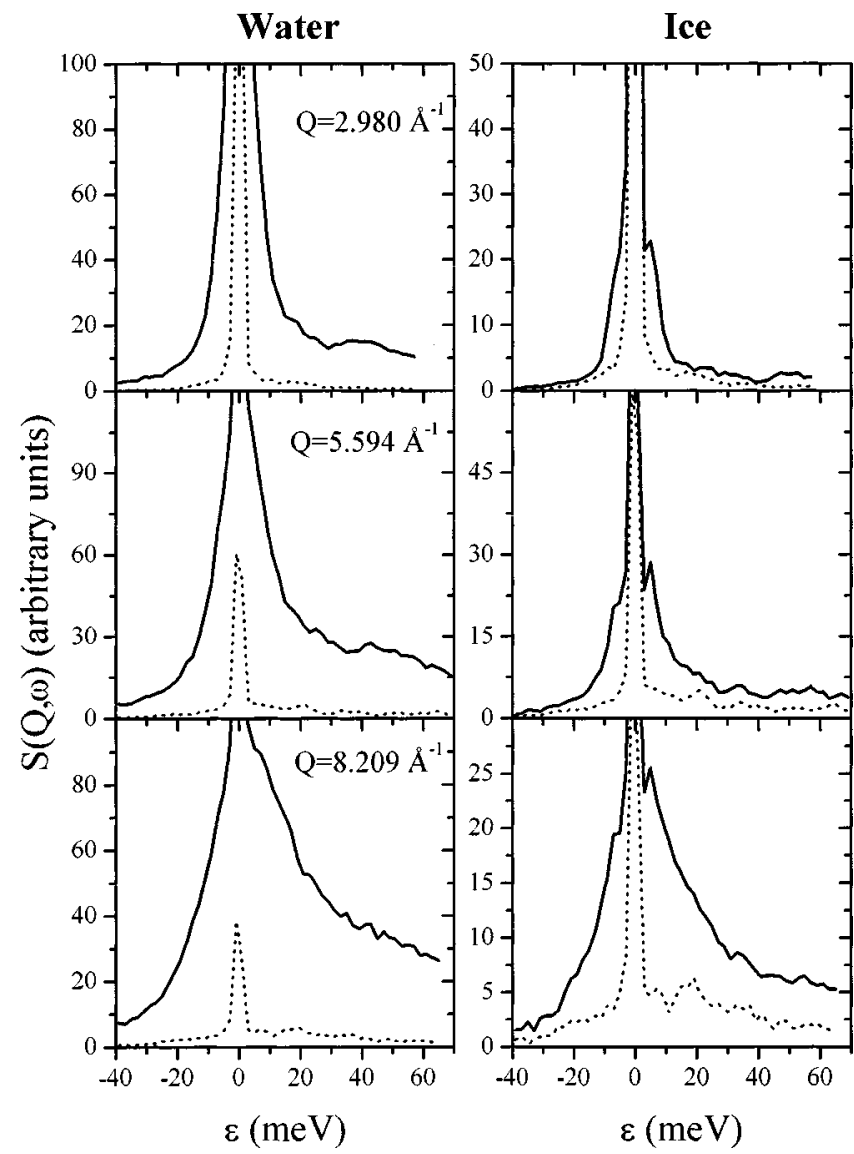

FIG. 1. Experimental $S(Q, \omega)$ for water (left column) and ice (right column) shown by the full lines. The dotted lines show the empty can results. Selected $Q$ values are representative of the regions in which a wider kinematic range is accesible.

which means to take Eq. (33) as valid for a given $Q$ value, thus allowing us to calculate $Z(\varepsilon)$ as an average over all the experimental $Q$ range to eliminate the coherent effects. ${ }^{11}$

The Debye-Waller factor can be written in terms of an average mean-square displacement $\left\langle u^{2}\right\rangle$ as

$$
2 W=\frac{\hbar^{2} Q^{2}}{2 M} \int_{-\infty}^{\infty} d \varepsilon u_{1}(Q, \varepsilon)=\frac{1}{3}\left\langle u^{2}\right\rangle Q^{2} .
$$

This equation is a normalization condition for the function $u_{1}(Q, \varepsilon)$, through the knowledge of $\left\langle u^{2}\right\rangle$,

$$
\frac{3 \hbar^{2}}{2 M} \int_{-\infty}^{\infty} d \varepsilon u_{1}(Q, \varepsilon)=\left\langle u^{2}\right\rangle .
$$

For any given $Q$ value an iterative procedure for calculating the multiphonon component is proposed. As a first guess we assume

$$
u_{1}^{(0)}(Q, \varepsilon)=\widetilde{S}(Q, \varepsilon),
$$

in which $\widetilde{S}(Q, \varepsilon)$ is the experimental dataset normalized according to Eq. (36) for the particular $Q$ value. We then calculate the multiphonon components from Eq. (34) and build the sum in Eq. (32) with a number of phonons $n$, which makes negligible the $n$th term [Eq. (34)]. Let us call

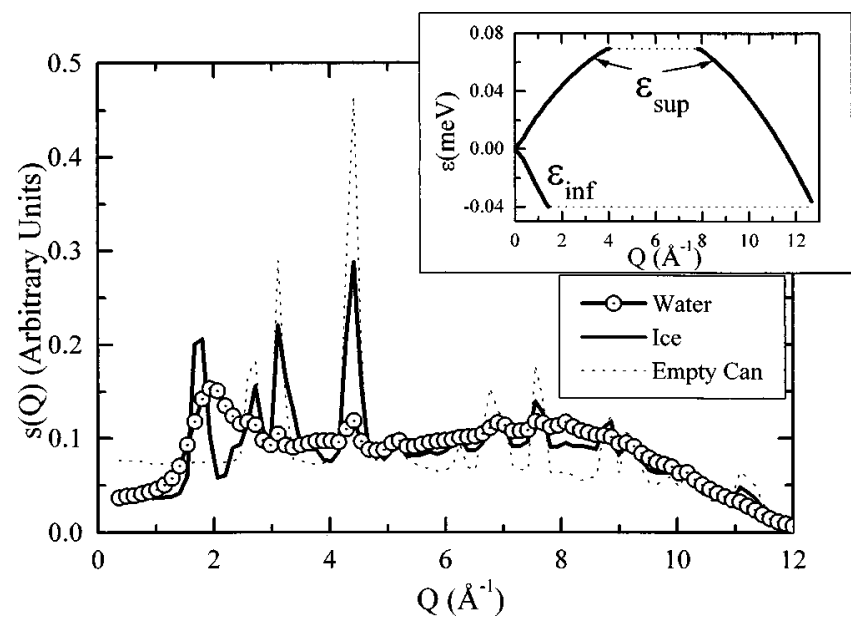

FIG. 2. Integral over all energy transfers in the allowed kinematic range of the experimental raw data for water, ice, and the empty cell. The data are normalized to the unit area and serve to illustrate the integration kernel of Eq. (13). The full lines in the inset correspond to kinematic limits, while dotted lines represent the limits of the datasets.

$v_{1}(Q, \varepsilon)$ and $v_{m}(Q, \varepsilon)$ the one-phonon and the multiphonon terms, respectively, if we define

$$
f_{M P}(Q, \varepsilon)=\frac{v_{m}(Q, \varepsilon)}{v_{1}(Q, \varepsilon)},
$$

then in the next iteration we can recalculate the one-phonon term as

$$
u_{1}^{(1)}(Q, \varepsilon)=\frac{u_{1}^{(0)}(Q, \varepsilon)}{1+f_{M P}(Q, \varepsilon)} .
$$

With this new one-phonon function we can recalculate the factor $f_{M P}(Q, \varepsilon)$ in an iterative way until no significant variations are observed in the multiphonon function.

\section{APPLICATION TO A REAL AND IMPORTANT CASE}

In this section we will show the results of the proposed correction procedure to determine the densities of states of water and ice. The reported experiment was performed at MARI, a direct geometry chopper spectrometer located at the ISIS pulsed neutron facility, Rutherford Appleton Laboratory (Oxfordshire, U.K.). The incident energy was $78.6 \mathrm{meV}$, with an energy resolution of about $1 \mathrm{meV}$. The sample was 99.99\% deuterated water contained in a hollow, cylindrical aluminum can placed perpendicularly to the incident beam with dimensions of $4.9 \mathrm{~cm}$ and $4.1 \mathrm{~cm}$ for the outer and inner diameters, respectively, and $6.3 \mathrm{~cm}$ in height. The thickness of the aluminum walls was $0.5 \mathrm{~mm}$ and the measurements were carried out at $270 \mathrm{~K}$ for the hexagonal $I h$ ice phase and $280 \mathrm{~K}$ for the liquid phase. For more details on the experiment, the reader is referred to Ref. 12.

In Fig. 1 experimental raw data are shown together with empty can results, for some selected values within the accessible kinematic range. In the case of ice, it is seen that at some particular regions the value of the experimental raw data is of the same order of magnitude as the empty can, which stresses the need for a careful can subtraction. In Fig. 
TABLE I. Input parameters for the synthetic model for water and ice employed in the Monte Carlo runs. The reader is referred to Sec. II B for reference.

\begin{tabular}{lcccc}
\hline \hline $\mathrm{D}_{2} \mathrm{O}$ (ice) & \multicolumn{5}{c}{} \\
\hline & Mode 1 & Mode 2 & Mode 3 & Mode 4 \\
$h \omega(\mathrm{meV})$ & 15 & 50 & 150 & 306 \\
$h \sigma(\mathrm{meV})$ & 1 & 21 & 18 & 19 \\
$M_{D}$ & 21.046 & 5.196 & 12.1 & 5.874 \\
$M_{O}$ & 24 & 213.9 & 290.3 & 179.3 \\
$\mathrm{D}_{2} \mathrm{O}($ water $)$ & & & & \\
\hline & & & & \\
$h \omega(\mathrm{meV})$ & Mode 1 & Mode 2 & Mode 3 & Mode 4 \\
$h \sigma(\mathrm{meV})$ & 15 & 50 & 150 & 306 \\
$M_{D}$ & 1 & 21 & 18 & 19 \\
$M_{O}$ & 40 & 5.20 & 12.1 & 5.87 \\
\hline \hline
\end{tabular}

2 we show plots of the integral [included in Eq. (14)]

$$
s_{\text {exp }}(Q)=\int_{\varepsilon_{\min }}^{\varepsilon_{\max }} s(Q, \varepsilon) d \varepsilon .
$$

Because the integral is constrained to the experimental kinematic range (which is shown in the inset), this magnitude is not exactly the structure factor $S(Q)$.

Monte Carlo simulations were performed over 30000 neutron histories for each iteration for each sample. Input data used in the simulations were the following: (a) Experi-

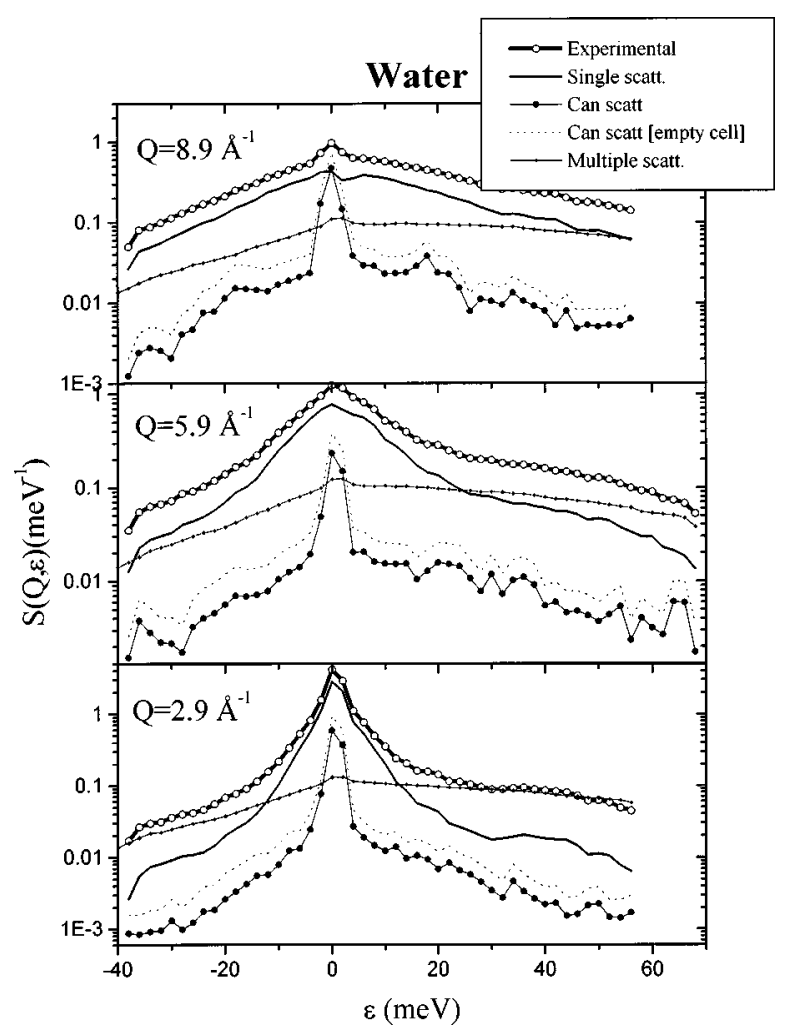

FIG. 3. Simulation results for water compared with the experimental data for three different $Q$ values. The dotted line without symbols shows the scattering from the empty cell, which has to be compared with the can scattering from the full cell.

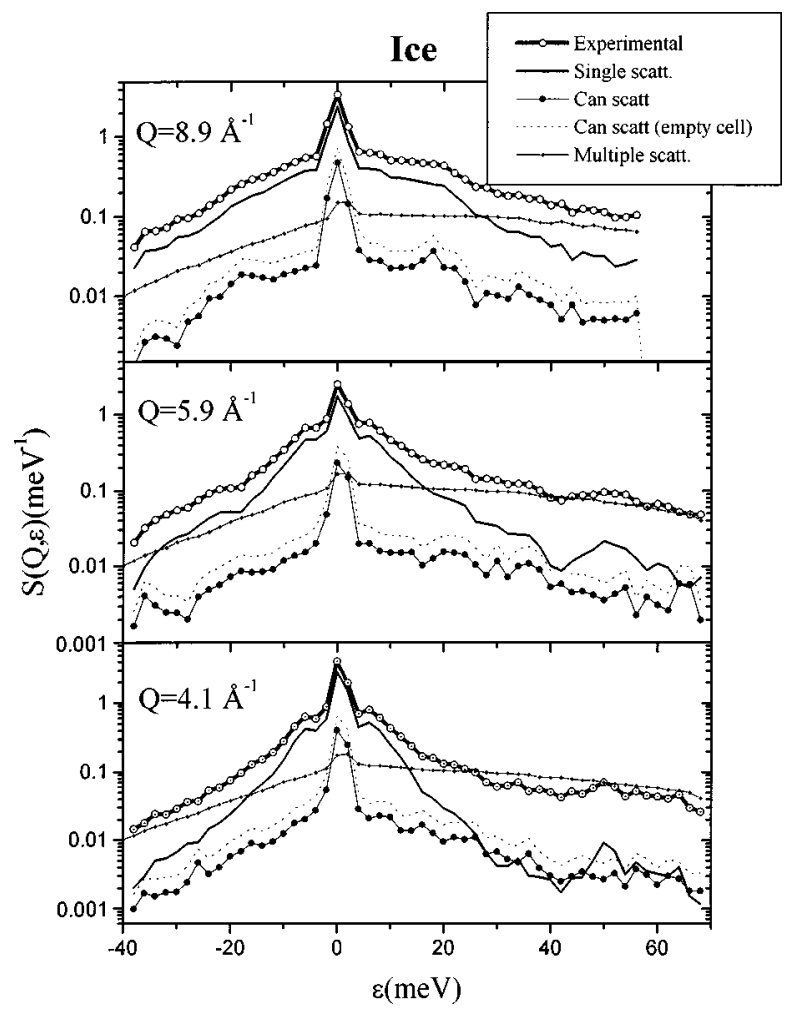

FIG. 4. The same results as in Fig. 3 for ice.

mental total and absorption cross sections of water (ice) in the range from $10^{-5}$ to $10^{3} \mathrm{eV}$; (b) Experimental total and absorption cross section of aluminum in the same range (can simulation); (c) Angular distributions for scattering in water (ice) taken from the experimental data, integrated as in Eq. (40); (d) Angular distributions for scattering in aluminum (can) taken from the experimental data, integrated as in Eq. (40); (e) The experimental raw data (for the first iteration) or corrected data from the preceding iteration; (f) Synthetic model input parameters for water (ice), summarized in Table I.

The geometry of the can was simulated as two concentric $1 \mathrm{~mm}$-thick aluminum cylinders, leaving a cylindric coronna of $41 \mathrm{~mm}$ inner diameter and $49 \mathrm{~mm}$ external diameter. The efficiency function of the detectors employed in the simulation was that of a typical ${ }^{3} \mathrm{He}$ tube, 1 inch diameter and 10 atm filling pressure. The cutoff weight for neutron histories was $10^{-6}$. Convergence of the multiple-scattering components was achieved after three iterations.

In Fig. 3 we show the results for each contribution from the simulations for water and in Fig. 4 for ice, compared with the experimental raw data, for three selected values of $Q$. Clearly, a large multiple-scattering correction has to be made in the tail of high energy transfers in the low $Q$ region. In fact, the multiple-scattering component is larger than the single one for energy transfers higher than some $12 \mathrm{meV}$. Although in this particular case no safe convergence of the iterative method is assured, it is seen that the sum of the calculated components reproduce fairly well the experimental data. The importance of the multiple-scattering component is still large in the tails at higher $Q$ values. A more difficult situation is found in the case of ice for low $Q$ values where the large multiple-scattering component and the 


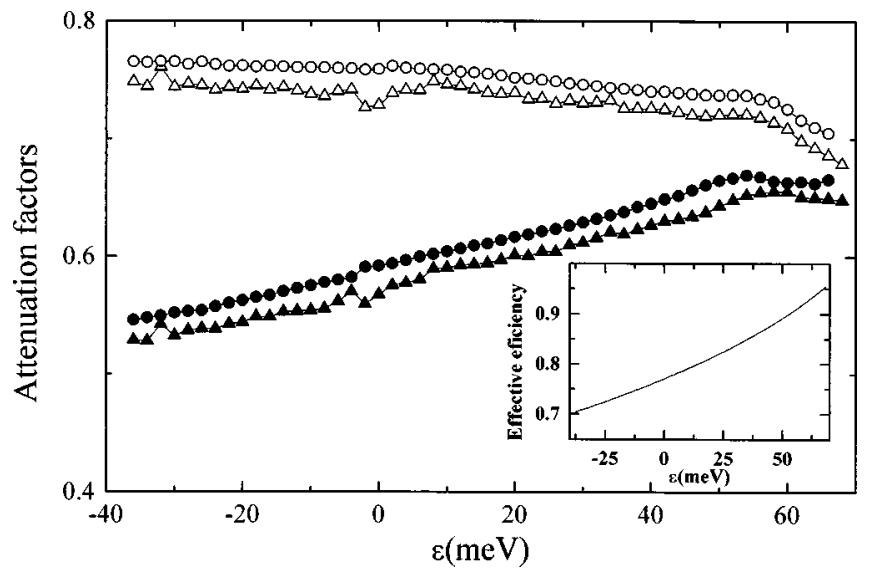

FIG. 5. Attenuation factors calculated including detector efficiency [as in Eq. (28), full symbols] and for the "black detector" [Eq. (13), hollow symbols]. Circles represent data for water and the triangles for ice. In the inset the effective detector efficiency for singly scattered neutrons is shown.

smaller inelasticity induce serious problems of convergence. For this reason, the multiple scattering is clearly overestimated in this region. This situation persists even at larger $Q$ values where the multiple scattering is dominant beyond some $20 \mathrm{meV}$. In spite of this, at $Q=5.9$ and $8.9 \AA^{-1}$ the sum of calculated components reproduce the experimental raw data quite satisfactorily. A special paragraph must be devoted to the scattering in the can. In Figs. 3 and 4 we show this component resulting from our sample + cell Monte Carlo simulations, as well as from the empty cell. It is clearly seen that the empty-cell result is $70 \%$ to $100 \%$ larger than the value from the sample + cell simulation. This magnitude is affected by attenuation in the sample as well as the multiplescattering interaction from the sample and the cell. This shows the nontriviality of empty can subtraction, which is more dramatic in the case of ice at low $Q$ and large energy transfer, where the empty cell and single-scattering effects are of the same order of magnitude.

The calculation of the attenuation factor is another subject that deserves a more exhaustive analysis. In Fig. 5 we compare the attenuation factor as calculated in Eqs. (13) and (28) for water and ice. It should be emphasized that these factors turned out to be almost independent of the number of iterations, and that no significant variation over the $Q$ range is observed, so we performed an average over all $Q$ values within the full kinematic range. The slight differences between water and ice are explained by their differences in density. From the graph, the importance of taking into account detector efficiency is apparent, because as demonstrated in Eq. (27), $H^{\prime}$ from Eq. (28) is the attenuation factor that links the experimental single-scattering component with the "true" single scattering, a fact that is often forgotten when direct Paalman-Pings corrections are applied. In the inset of Fig. 5 is shown the detector efficiency for the experimental range of energy transfers. This efficiency was calculated for single-scattering processes, although no major differences with that calculated from multiple-scattering processes was observed due to the small energy dispersion in each effective energy channel. The situation is rather differ-

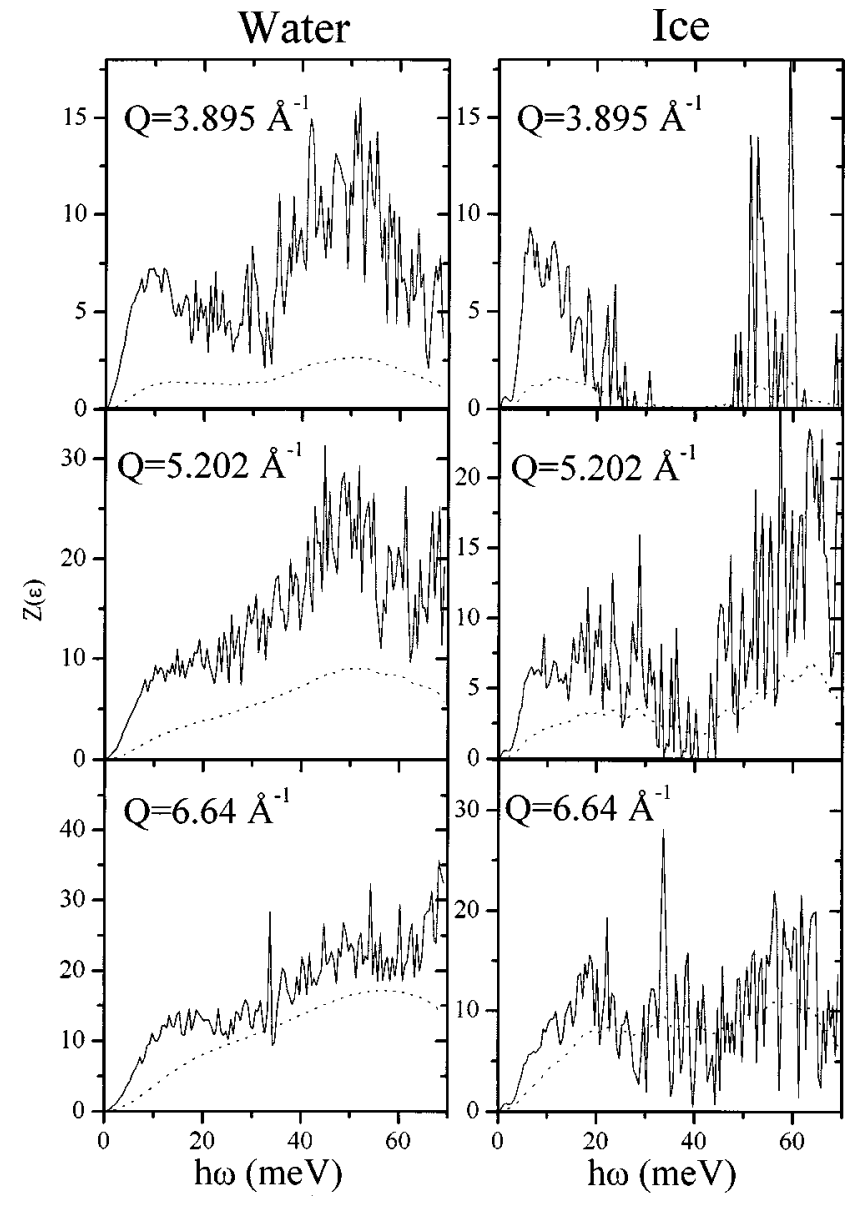

FIG. 6. Calculated densities of states $Z(\varepsilon)$ after multiphonon correction for water and ice for three $Q$ values (full line). The dotted line shows the contribution of multiphonon effects to the experimental data.

ent in diffraction experiments, ${ }^{10}$ where for a $Q$ bin the energy distribution for singly and multiply scattered neutrons are quite different.

Multiphonon effects are illustrated in Fig. 6, where we show the densities of states $Z(\varepsilon)$ resulting from the iterative processes that converged after three steps in every case. Ten phonons were enough to assure the convergence of the multiphonon components, which are shown in the same figure multiplied by the factor $\varepsilon /(n(\varepsilon)+1)$, to make them comparable with $Z(\varepsilon)$ in the same plot. The scale employed in ordinates is such that the normalization condition for $u_{1}(Q, \varepsilon)$ from Eq. (36) for a value of $\left\langle u^{2}\right\rangle=0.0458 \AA^{2}$ is met. ${ }^{12}$ It is observed that the single and multiphonon components are comparable at $Q=6.64 \AA^{-1}$, which is at the middle of the kinematic range, and become dominant for higher $Q$ values.

Finally, in Fig. 7 we present the densities of states that result after the application of the corrective procedure described in this paper. As usual, to compensate for the coherent effects, an average over $Q$ was performed, although some care was exercised in the choice of the $Q$ range. In the case of ice, a range from 0.88 to $6.64 \AA^{-1}$ was chosen, and it was observed that in order to correctly account for all the vibrational modes it was necessary to average on a $\mathrm{Q}$ range as wide as possible, and at the same time to avoid too high $Q$ values where multiphonon corrections are severe. In the case 


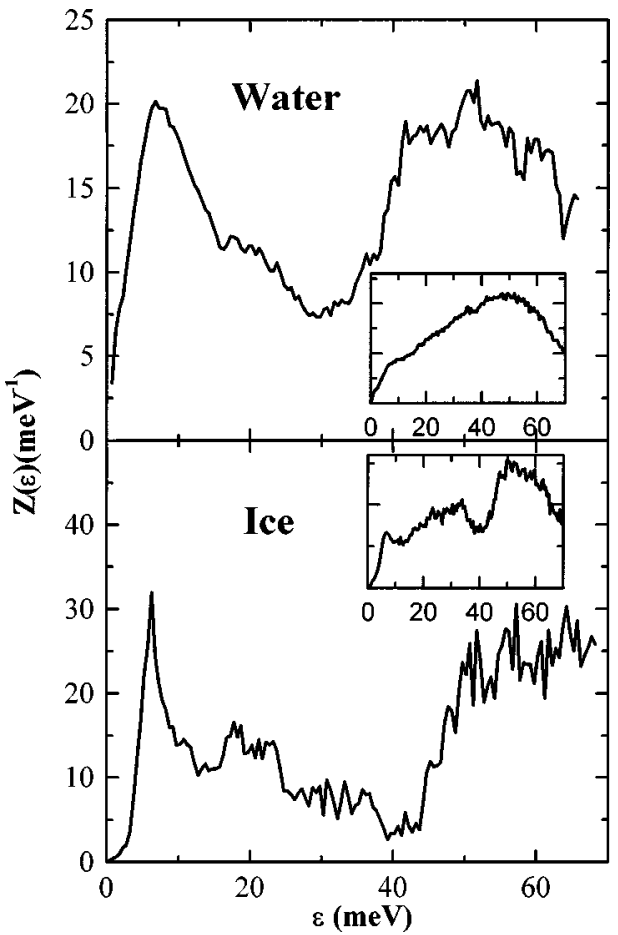

FIG. 7. Final densities of states for water and ice obtained after all the corrections presented in this paper. Averages over a range of 0.88 to $6.64 \AA^{-1}$ for ice and 0.88 to $4.3 \AA^{-1}$ for water were performed in the corrected $S(Q, \omega)$. In the insets of each frame we show the densities of states that would have been obtained if no multiple scattering and multiphonon corrections were performed.

of water, the chosen range was from 0.88 to $4.3 \AA^{-1}$. It has to be noted that the quantities of interest here take into account that part of the liquid dynamics that can be regarded as arising from vibrational modes takes place at finite frequencies (i.e., stable modes in the instantaneous normal mode terminology). ${ }^{15}$ Other kind of motions, that is, those of stochastic nature, such as molecular long-range translations and single-particle reorientations that occur at zero frequencies, will also contribute to the low-frequency density of states, and a proper evaluation of this would require extrapolation procedures to $\mathrm{Q} \rightarrow 0$ such as those described in Ref. 16. To emphasize the need of an accurate procedure for mutiple scattering and multiphonon corrections, in the insets of Fig. 7 we show the densities of states obtained directly from the raw data without any correction. We can readily conclude that if no corrections are performed, the results can show features not present in the real functions, and the real shape can be blurred or hidden.

A final comment on the extraction of the density of states was pointed out recently by Taraskin and Elliot ${ }^{14}$ in the analysis of multiatomic systems, which is related to that obtained from $S(Q, \omega)$ through a correction factor, that in the case of water is not negligible, ranging from 0.34 to 3.63 according to Eqs. (31) and (32) in that paper. As proposed by the authors, a numerical simulation on the system that leads to the desired function is indispensable.

\section{SUMMARY AND CONCLUSIONS}

In this paper we analyzed an inelastic neutron-scattering experiment performed on a state-of-the-art neutron spectrom- eter, which yielded data of good resolution and counting statistics for deuterated water and ice. A fairly careful and detailed correction procedure is, however, needed to obtain the desired information from the measured distributions of neutron counts.

A correction procedure was presented that is based upon the description of the scattering system in terms of the Synthetic Model and a Monte Carlo strategy for which the former provides rather efficient kernels. Such a combined strategy enables the correction for multiple scattering and sample container to be performed in a controlled way. It also serves to account for the attenuation of the neutron beam by single-scattering processes. Emphasis was made in the effect introduced by the detector efficiency, which has to be known beforehand in a detailed manner. This effect was included in the primary attenuation factor together with the neutron attenuation calculated from experimental neutron total cross sections as a function of energy. Empty-cell runs are needed to measure the shape of the inelastic spectrum of the container to be included in the Monte Carlo code, but a large error can be introduced if a direct subtraction is made on the basis of such experimental data only. Even in the present experiment where this effect is relatively small, it is seen that for ice at large energy transfers an incorrect can subtraction can lead to fairly notorious effects, and this can be of decisive importance in the case of experiments with pressure cells. It is worth mentioning that even counting upon a reliable correction tool for multiple-scattering processes such as the present one, the premise of reducing experimentally those processes as much as possible remains a valid motto. It will guarantee the convergence of the iterative process down to a reasonable solution.

A method for correcting for multiphonon processes was described and employed to obtain the density of states. Contrary to the case of multiple scattering, multiphonon effects cannot be reduced experimentally, except by lowering the temperature as much as possible. Naturally, such a procedure is not the one of choice if temperature effects are the topic to be investigated, such as the anharmonic effects on the vibrational spectra. The procedure outlined here allows a fast convergence that permits us to obtain a reliable approximation to the density of states.

As a test of the importance of multiphonon and multiplescattering effects at large energy transfers, it may suffice to compare the present data for liquid water with previous derivations of the spectra from the anti-Stokes side of coldneutron spectra. ${ }^{17}$ As seen from Fig. 7 of Ref. 17, as well as from a number of subsequent experiments, an unphysically large band centered at about $70 \mathrm{meV}$ is reported. Its integrated intensity, which is many times larger than that of the spectrum below $30 \mathrm{meV}$, shows that it cannot come from single-phonon processes (it would run counter to the equipartition principle otherwise).

Estimates of the spectral frequency distribution of liquid water have been reported many times as a result of molecular dynamics calculations using a variety of intermolecular potential functions. However, most of the quantities which appeared in the literature since the pioneering paper on this topic by Rahman and Stillinger, ${ }^{18}$ refer to motions of the molecular center of mass or to the frequency spectrum for proton motions, a quantity to be compared with that acces- 
sible through incoherent inelastic neutron scattering. This hampers a detailed comparison with results derived in this work, although some comments, on semiquantitative grounds are worth making. The spectrum for total proton motions (a combination of reorientations and motion of the molecular center-of-mass) shown in the original paper (Fig. 32 of Ref. 18) is strongly peaked about $53 \mathrm{meV}$ and extends up to $\approx 160 \mathrm{meV}$ without showing hints of a gap. In contrast, more recent estimates ${ }^{15}$ depict, after a normal-mode analysis of quenched configurations (i.e., those modes of a liquid configuration that are quenched and subsequently allowed to relax to a local minimum by means of steepest descents), a far richer structure in the spectrum. In particular, a gap is seen at about $49-50 \mathrm{meV}$ separating a highfrequency band assignable to molecular rotations that extends up about $100 \mathrm{meV}$ (notice that a strong isotopic effect will affect this band as a consequence of the $H / D$ substitu- tion) and a low-frequency region showing a well-defined peak at about 6-8 $\mathrm{meV}$ and additional structure peaking at about $37 \mathrm{meV}$. Even if the structure appearing in such a spectrum will be strongly smeared if the calculation is carried at a finite temperature (i.e., will include mass diffusion and finite-lifetime effects), the basic ingredients seen in the experiment and simulation seem to agree on semiquantitative grounds.

As regards the comparison of present results with spectra for hexagonal ice at high temperature, up to the authors' knowledge, most detailed calculations of the full frequency spectrum have focused themselves into the harmonic dynamics (see, for instance, Ref. 19). This makes a comparison rather difficult to perform since anharmonic effects at finite temperatures are known to be remarkably large, ${ }^{20}$ which would result in significant frequency shifts and rather large broadening of some peaks.
${ }^{1}$ A series of international conferences on data treatment for experiments carried out on radiation-scattering sources have been convened in recent years (unpublished).

${ }^{2}$ E. Duval, N. García, A. Boukenter, and J. Serrughetti, J. Chem. Phys. 99, 2040 (1993); T. Achibat, A. Boukenter, and E. Duval, ibid. 99, 2046 (1993).

${ }^{3}$ H. H. Paalman and C. J. Pings, J. Appl. Phys. 31, 2635 (1962).

${ }^{4}$ V. F. Sears, Adv. Phys. 24, 1 (1975).

${ }^{5}$ J. R. D. Copley, Comput. Phys. Commun. 7, 289 (1974); J. R. D. Copley, P. Verkerk, A. A. Van Well, and H. Fredrikze, ibid. 40, 337 (1986).

${ }^{6}$ J. Dawidowski, G. J. Cuello, and J. R. Granada, Nucl. Instrum. Methods Phys. Res. B 82, 459 (1993).

${ }^{7}$ J. R. Granada, Phys. Rev. B 31, 4167 (1985).

${ }^{8}$ Some of these applications can be found in J. R. Granada, V. H. Gillette, and R. E. Mayer, Phys. Rev. A 36, 5594 (1987); 36, 5585 (1987); 36, 5594 (1987); J. R. Granada, J. Dawidowski, R. E. Mayer, and V. H. Gillette, Nucl. Instrum. Methods Phys. Res. A 261, 573 (1987); J. R. Granada and V. H. Gillette, J. Neutron Res. (to be published).

${ }^{9}$ J. Dawidowski, J. R. Granada, R. E. Mayer, G. J. Cuello, V. H. Gillette, and M. C. Bellissent-Funel, Physica B 203, 116 (1994).

${ }^{10}$ J. Dawidowski, J. R. Granada, G. J. Cuello, and V. H. Gillette,
Physica B 213\&214, 616 (1995).

${ }^{11}$ J. Dawidowski, F. J. Bermejo, R. Fayos, R. Fernández Perea, S. M. Bennington, and A. Criado, Phys. Rev. E 53, 5079 (1996).

${ }^{12}$ F. J. Bermejo, M. Alvarez, S. M. Bennington, and R. Vallauri, Phys. Rev. E 51, 2250 (1995).

${ }^{13} \mathrm{~S}$. W. Lovesey, Theory of Neutron Scattering from Condensed Matter (Clarendon, Oxford, 1987), p. 63.

${ }^{14}$ S. N. Taraskin and S. R. Elliot, Phys. Rev. B 55, 117 (1997).

${ }^{15}$ A description of the high-frequency dynamics in terms of normal modes for liquid water is given in F. Sciortino and S. Sastry, J. Chem. Phys. 100, 3881 (1994).

${ }^{16}$ P. A. Egelstaff, An Introduction to the Liquid State (Oxford Science Publications, New York, 1992), p. 243.

${ }^{17}$ S. H. Chen, in Hydrogen-Bonded Liquids, edited by J. C. Dore and J. Teixeira (Kluwer, Dordrecht, 1991), p. 310.

${ }^{18}$ A. Rahman and F. H. Stillinger, J. Chem. Phys. 55, 3336 (1971). See also, M. Cho, G. R. Feming, S. Saito, I. Ohmine, and R. M. Stratt, ibid. 100, 6672 (1994).

${ }^{19}$ A. Criado, F. J. Bermejo, M. García-Hernandez, and J. L. Martínez, Phys. Rev. E 47, 3516 (1993).

${ }^{20}$ For an estimate of the anharmonic effects in ice at high temperature, see D. D. Klug, E. Whalley, E. C. Svensson, J. H. Root, and V. F. Sears, Phys. Rev. B 44, 841 (1991). 